\title{
Papilomavirus y Factores asociados a Neoplasia Intraepitelial Cervical de Alto Grado en Cauca, Colombia
}

\author{
Carlos H. Sierra Torres ${ }^{1}$, Maria P. Acosta Aragón ${ }^{2}$ y Leonora Orejuela Aristizabal ${ }^{3}$ \\ ${ }^{1}$ Licenciado en Biología, Ph.D. Ciencias Biomédicas. Laboratorio de Genética Humana, Departamento \\ de Ciencias Fisiológicas, Facultad de Ciencias de la Salud, Universidad del Cauca, Popayán, Colombia. \\ E-mail: hsierra@unicauca.edu.co \\ ${ }^{2}$ Médica. Especialista en Ginecologia y Obstetricia. Departamento de Ginecología y Obstetricia, Facultad \\ de Ciencias de la Salud, Universidad del Cauca, Popayán, Colombia. \\ E-mail: macosta@unicauca.edu.co \\ ${ }^{3}$ Bióloga. Laboratorio de Genética Humana, Departamento de Ciencias Fisiológicas, Facultad de \\ Ciencias de la Salud, Universidad del Cauca, Popayán, Colombia. E-mail: leorejuela@hotmail.com
}

Recibido 18 Julio 2005/Enviado para Modificación 19 Octubre 2005/Aceptado 12 Enero 2006

\section{RESUMEN}

Objetivo Evaluar el papel de los principales factores de riesgo asociados a lesiones intraepiteliales escamosas de alto grado en mujeres del Departamento del Cauca, Colombia.

Metodología Después de la firma de un consentimiento informado, las mujeres fueron encuestadas para la obtención de datos socio-demográficos y de estilo de vida. Adicionalmente, de cada mujer se obtuvo una biopsia cervical para diagnóstico histopatológico (casos) y una muestra de células exfoliadas (casos y controles) para posterior extracción de ADN y detección de papilomavirus (VPH) por reacción en cadena de la polimerasa (PCR). Un total de 98 casos y 109 controles, apareados por edad y procedencia, fueron reclutados para este estudio.

Resultados El estudio confirma la asociación entre VPH y el riesgo de neoplasia cervical (OR=19,0; IC95\%=8,20-44,2). Los datos sugieren que la multiparidad $(\mathrm{OR}=4,1 ; \quad \mathrm{IC} 95 \%=1,62-10,6)$ y la exposición a carcinógenos presentes en el humo de leña $(O R=7,3$; IC95\%=3,00-19,4) son importantes co-factores de riesgo dada la presencia de VPH.

Conclusiones Estos resultados brindan información valiosa a las instituciones de salud pública para desarrollar mejores programas de promoción y prevención de neoplasia cervical.

Palabras Clave: Neoplasia cervical intraepitelial, factores de riesgo, Papillomavirus Humano, paridad, Colombia (fuente: DeCS, BIREME)

\section{ABSTRACT}




\section{Papillomavirus and factors associated with high-risk, cervical intra- epithelial neoplasia in Cauca, Colombia}

Objective Evaluating the role of the main factors associated with high-risk cervical intraepithelial neoplasia in women from the Cauca Department in Colombia.

Methodology After signing a consent-form, women were interviewed to obtain socio-demographic and lifestyle data. A cervical biopsy was performed on each woman for histopathological diagnosis (cases) and a sample of exfoliated cells (cases and controls) to extract DNA for HPV detection by polymerase chain reaction. A total of 98 cases and 109 controls, matched by age and ethnicity, were recruited for the study.

Results The study confirmed association between HPV and the risk of cervical neoplasia $(\mathrm{OR}=19.0 ; 95 \% \mathrm{Cl}=8.20-44.2)$. The data suggested that multiparity $(\mathrm{OR}=4.1 ; 95 \% \mathrm{Cl}=1.62-10.6)$ and exposure to carcinogens present in wood-smoke (OR=7.3; $95 \% \mathrm{Cl}=3.00-19.4)$ are important co-factors for cervical neoplasia given the presence of HPV.

Conclusions These results provide valuable information for public health institutions to develop better cervical neoplasia prevention programmes.

Key Words: Cervical intraepithelial neoplasia, risk factor, human papillomavirus, parity, Colombia (source: $M e S H, N L M$ ).

$\mathrm{S}$ egún la Agencia Internacional para la Investigación en Cáncer (IARC), los carcinomas del tracto anogenital, particularmente el cáncer de cuello uterino (CCU), representan el 12 \% de todos los canceres en las mujeres; el CCU ocupa el segundo lugar en incidencia a nivel mundial (52 000 nuevos casos/año), después del cáncer de seno (1). En el año 2000, al menos 76000 casos incidentes de CCU y 30000 muertes se estimaron para América Latina en general, representando 16 y $13 \%$ del total del mundo, respectivamente (2). En Colombia, el CCU también constituye la segunda causa de mortalidad en mujeres (16/100 000) y registra una incidencia de 32 por cada 100000 mujeres (3). Según el Instituto Nacional de Cancerología, la mayoría de las neoplasias malignas detectadas en cérvix se encuentran en estados avanzados (III y IV) (4). En el Cauca, según la Dirección Departamental de Salud, el CCU es la primera causa de mortalidad por cáncer en mujeres, registrando un promedio de 38 muertes por año en la última década (datos no publicados).

Desde la identificación de las lesiones condilomatosas como precursoras de cáncer en el cuello uterino en la década de los setenta (5), se han realizado varias investigaciones para conocer el papel de la actividad sexual en el riesgo de $\mathrm{CCU}$, indicando que las mujeres con relaciones sexuales a una 
edad temprana o varios compañeros sexuales, tendían a presentar un mayor riesgo de desarrollar CCU (6). Con el desarrollo de técnicas en biología molecular para detectar la presencia de ADN del virus del papiloma humano (VPH) en muestras citológicas durante la década de los ochenta, se pudo establecer el papel del VPH de alto riesgo en la etiología del CCU (7). Posteriormente, estudios epidemiológicos multicentro conducidos en la década de los noventa, permitieron demostrar la asociación causal entre infecciones con VPH y el desarrollo de CCU (8). Con la evidencia científica acumulada, hoy en día, la infección con VPH ha sido propuesta como la primera "causa necesaria” para el desarrollo de CCU (9). Sin embargo, no todas las mujeres infectadas con VPH desarrollan cáncer invasor, existiendo esta infección con frecuencia en mujeres sanas. Por lo tanto, otros co-factores deben ser necesarios para la progresión de la carcinogénesis en el cuello uterino (10).

El objetivo de este artículo es reportar la asociación del VPH y otros cofactores con el riesgo para desarrollar lesiones intraepiteliales escamosas de alto grado en mujeres del departamento del Cauca. Además del alto riesgo asociado a la infección con VPH, los resultados muestran una novedosa asociación entre la exposición al humo de leña en la cocina, la cual es una práctica muy común en las mujeres de las zonas rurales del Cauca, y el incremento en el riesgo de este tipo de lesiones.

\section{MATERIALES Y MÉTODOS}

Población de estudio y muestreo

Este estudio prospectivo de casos y controles (1:1) se realizó en una población femenina del departamento del Cauca entre enero de 2002 y diciembre de 2003. Los casos $(n=98)$ se definieron como aquellas mujeres con lesiones intraepiteliales escamosas de alto grado (NIC II, III y carcinoma in situ) y se seleccionaron entre las mujeres remitidas al Centro de Patología Cervico-Uterina (CPCU) del Hospital Susana López de Valencia (HSLV) de la Ciudad de Popayán. Los controles $(n=109)$ fueron definidos como mujeres con un cuello uterino sano, con citología "normal" en el último año, sin historia previa de tratamiento por infección con VPH (Ej. crioterapia) y se colectaron en los servicios de citología del Hospital Nivel I del Bordo, Hospital Nivel I de Piendamó y el HSLV y Profamilia en Popayán.

Después de la firma voluntaria del consentimiento informado, según lo dispuesto en la Resolución No. 8430 de 1993 del Ministerio de Salud, las participantes fueron entrevistadas a través de un cuestionario para colectar las variables de interés. Posteriormente, se obtuvo una biopsia cervical para 
diagnóstico histopatológico de la lesión (casos) y una muestra de células exfoliadas del cuello uterino (casos y controles) para posterior extracción de ADN y detección de VPH por reacción en cadena de la polimerasa (PCR).

Los controles se parearon con los casos, según edad ( \pm 5 años) y procedencia (urbana o rural). Otras variables como estrato social, ingresos, educación, etc. no se tuvieran en cuenta para parear. Los criterios de exclusión del estudio fueron exposición a drogas quimo-terapéuticas, enfermedades crónicas, historia familiar de cáncer, que no se deseara participar en el estudio o que su estado de salud no se lo permitiera.

Extracción de ADN y detección de ADN viral

El ADN genómico fue extraído de células exfoliadas cervicales utilizando la técnica por Chelex (SIGMA, No. C7901) (11). Para la detección del VPH se utilizo una técnica previamente estandarizada y utilizada por el grupo de investigación (12). En resumen, la reacción de PCR (50 $\mu \mathrm{L})$ consistió de 100 ng de ADN, 35 pmol de cada cebador: 1s-TAGGGTTACATTGCTATGAG, 3s-ATGGCTGATCCWGMAGGTAC,1as-TTCCACTTCAGWAYWGCCA TA. Se agregó $200 \mu \mathrm{mol}$ de dNTPs, $5 \mu \mathrm{L}$ de 10X PCR buffer, 1,5 mM MgCl2, y 2 U de Taq DNA polymerase (No. M1865, Promega, Madison, WI, USA). Las condiciones de PCR consistieron de 4 min a $94^{\circ} \mathrm{C}$, seguido por 35 ciclos de $94^{\circ} \mathrm{C}$ por $40 \mathrm{seg}, 56^{\circ} \mathrm{C}$ por $30 \mathrm{seg}, 72^{\circ} \mathrm{C}$ por $30 \mathrm{seg}$, y un paso final de $72^{\circ} \mathrm{C}$ por $10 \mathrm{~min}$. Los productos de amplificación fueron separados por electroforesis usando un gel de 3:1 Nusieve Agarose al 2,5 \% corriendo a 80 voltios por $45 \mathrm{~min}$. La amplificación de bandas a 670 o $420 \mathrm{pb}$ indicó la presencia de VPH de bajo o alto riesgo, respectivamente. Todas las reacciones incluyeron controles internos de amplificación utilizando cebadores específicos para $\beta$-globina, como se describe en la literatura (12).

\section{Análisis estadístico}

El análisis de datos se realizo en el paquete estadístico SPSS versión 10 (SPSS Inc., Chicago, IL, EUA). Las variables continuas se expresaron con la media \pm desviación estándar y las variables discretas se expresaron en frecuencias y proporciones. Para estimar el riesgo relativo, se calculo los OR y los intervalos de confianza al $95 \%$, utilizando un modelo de regresión logístico múltiple (12). Adicionalmente, los OR fueron ajustados por las potenciales variables de confusión VPH (presencia vs. ausencia), edad (variable continua), lugar de procedencia (urbano vs. rural) y nivel educativo (ninguno, primaria, secundaria o superior). 


\section{RESULTADOS}

Un total de 207 mujeres fueron reclutadas para este estudio (98 casos y 109 controles). Los diagnósticos histopatológicos de los casos correspondieron a 16 (16 \%) NIC II, 35 (36 \%) NIC III y 47 (48 \%) carcinomas in situ. Como se indica en la Tabla 1, la edad promedio de los casos fue 44,2 $\pm 14,3$ años. Un total de 47 (48 \%) casos se encontraron en edades entre los 18 y 40 años, periodo considerado como "adecuado" para la reproducción. La mayoría de los casos procedían de áreas rurales (78 \%). El 52 \% de los casos reportaron no poseer ningún tipo de formación académica en comparación a un 25 \% en los controles. No se observaron diferencias significativas en las variables de apareamiento (edad y procedencia) entre los casos y los controles.

Tabla 1. Características demográficas de la población de estudio

\begin{tabular}{|c|c|c|c|}
\hline Características & $\begin{array}{c}\text { Casos } \\
\text { n (\%) }\end{array}$ & $\begin{array}{c}\text { Controles } \\
n(\%)\end{array}$ & p \\
\hline Total & $98(100)$ & $109(100)$ & N.A. \\
\hline $\begin{array}{l}\text { Edad (años) } \\
\text { Media } \pm \text { DS } \\
\leq 30 \\
31-40 \\
41-50 \\
>50\end{array}$ & $\begin{array}{c}44,2 \pm 14,3 \\
16(16) \\
31(32) \\
18(18) \\
33(34)\end{array}$ & $\begin{array}{c}42,9 \pm 10,1 \\
16(15) \\
31(28) \\
35(32) \\
27(25)\end{array}$ & $\begin{array}{l}0,451^{a} \\
0,140^{b}\end{array}$ \\
\hline $\begin{array}{l}\text { Procedencia } \\
\text { Urbana } \\
\text { Rural }\end{array}$ & $\begin{array}{l}22(22) \\
76(78)\end{array}$ & $\begin{array}{l}32(29) \\
77(71)\end{array}$ & $0,272^{b}$ \\
\hline $\begin{array}{l}\text { Consumo de cigarrillo } \\
\text { Si } \\
\text { No }\end{array}$ & $\begin{array}{c}3(3) \\
95(97)\end{array}$ & $\begin{array}{c}3(3) \\
106(97)\end{array}$ & $1,000^{b}$ \\
\hline $\begin{array}{l}\text { Consumo de alcohol } \\
\text { Nunca } \\
1-2 \text { veces/mes } \\
\text { 1-2 veces/año }\end{array}$ & $\begin{array}{l}38(39) \\
16(16) \\
44(45)\end{array}$ & $\begin{array}{l}40(37) \\
18(16) \\
51(47)\end{array}$ & $0,951^{b}$ \\
\hline $\begin{array}{l}\text { Nivel educativo } \\
\text { Ninguno } \\
\text { Primaria } \\
\text { Secundaria } \\
\text { Superior }\end{array}$ & $\begin{array}{c}51(52) \\
40(41) \\
6(6) \\
1(1)\end{array}$ & $\begin{array}{l}27(25) \\
49(45) \\
19(17) \\
14(13)\end{array}$ & $0,001^{b}$ \\
\hline
\end{tabular}

La Tabla 2 muestra que la infección con VPH fue un factor de riesgo altamente significativo, estando presente en $91 \%$ de los casos en comparación a un 36 \% en los controles. Después de ajustar en el modelo de regresión lo- 
gística múltiple con las potenciales variables de confusión, el riesgo fue 19 veces mayor dada la presencia de VPH (Tabla 2).

Tabla 2. Principales factores y riesgos asociados a neoplasia cervical en el Cauca

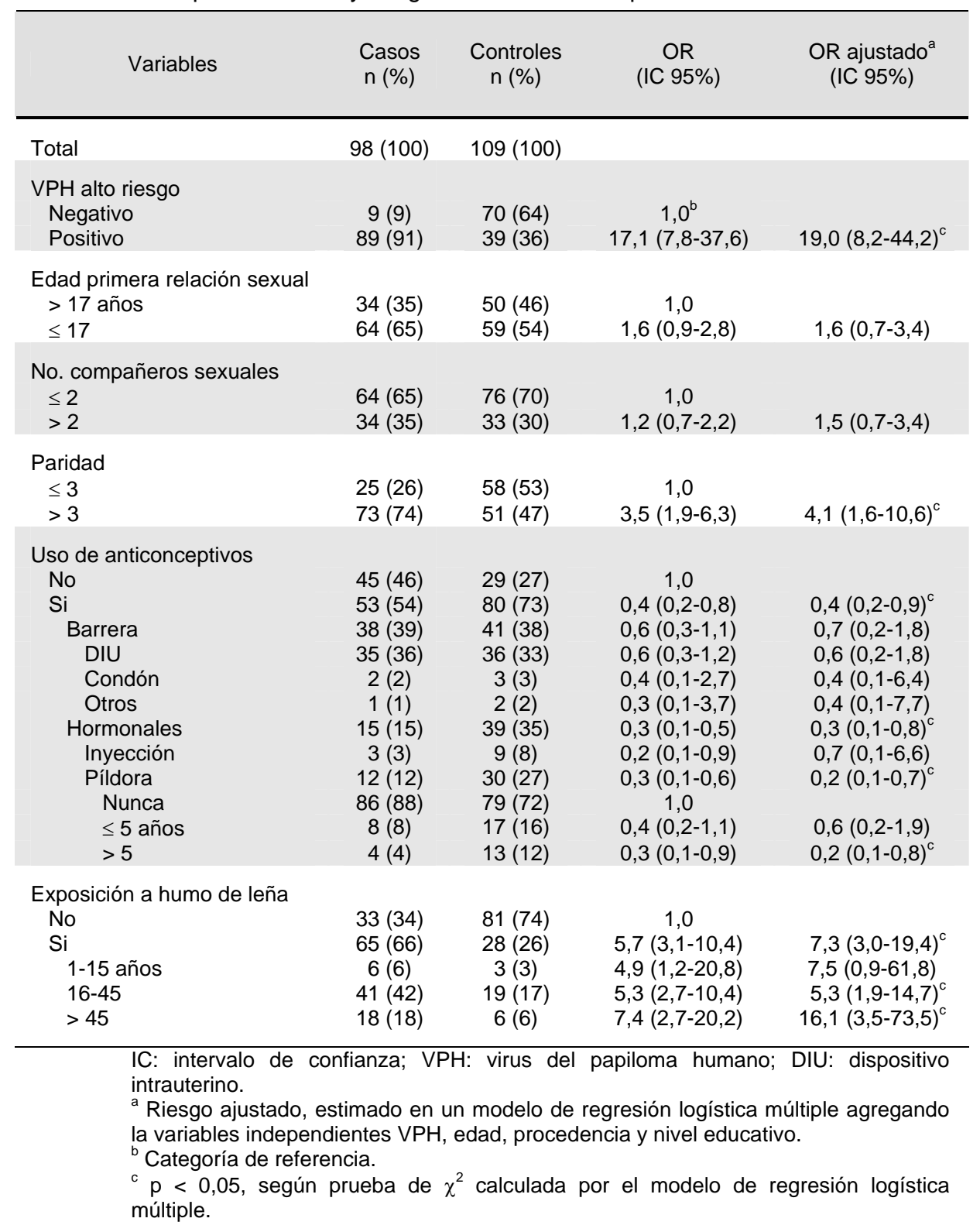


Como se indica en la Tabla 2, tener la primera relación sexual a una edad menor o igual a 17 años $(\mathrm{OR}=1,6)$ o más de 2 compañeros sexuales $(\mathrm{OR}=1,5)$ no afectó significativamente el riesgo, incluso después de ajustar por las variables de confusión. En un análisis posterior (datos no mostrados), la diferencia entre edad a la primera relación sexual $(17,2 \pm 3,6$ vs. 17,6 $\pm 3,7$, $\mathrm{p}=0,476)$ y el número de compañeros sexuales $(2,5 \pm 2,6$ vs. $2,6 \pm 2,9, \mathrm{p}=$ $0,770)$ no fue estadísticamente significativa entre casos y controles, respectivamente. Sin embargo, se observó que los casos menores o iguales 30 años tuvieron su primera relación sexual a una edad significativamente menor que la de los controles $(15,3 \pm 1,8$ vs. $17,9 \pm 3,8, p=0,020$, respectivamente).

La mayoría de los casos (74 \%) reportaron ser multíparas (> 3 partos), lo cual estuvo asociado con un riesgo de 4,1 veces mayor (Tabla 2). En un análisis posterior (datos no mostrados), el número promedio de partos en la población de casos fue significativamente mayor en relación a los controles (5,3 $\pm 2,6$ vs. $3,8 \pm 2,5, p<0,001$, respectivamente).

El uso de anticonceptivos fue mayor en los controles (73\%) en comparación con los casos (54\%), lo que redujo el riesgo en un 60 \%, incluso después de ajustar por las variables de confusión (Tabla 2). Al analizar el riesgo según el tipo de anticonceptivo, los métodos de barrera tendieron a disminuir el riesgo, pero este no fue significativo. Los métodos hormonales también tendieron a reducir el riesgo, pero solamente la píldora fue asociada a una reducción significativa en el riesgo; en particular, en mujeres que la habían usado por más de 5 años $(\mathrm{OR}=0,2)$.

El uso de leña como combustible en la cocina fue mayor en los casos (66 \%) en comparación a los controles (26 \%), generando un riesgo 5,7 veces mayor en los casos, el cual aumentó significativamente hasta 7,3 una vez se ajustó por las variables de confusión (Tabla 2). Al analizar el promedio de años que las mujeres habían estado expuestas al humo de leña (datos no mostrados), no se encontró una diferencia estadísticamente significativa entre casos y controles $(34,3 \pm 14,7$ vs. $33,7 \pm 10,6, p=0,860$, respectivamente). Sin embargo, al categorizar las mujeres por tiempo de exposición (Tabla 2), se observó un aumento en el riesgo dependiente del tiempo de exposición, el cual se mantuvo después del ajuste por variables de confusión para las categorías 16-45 y mayores de 45 años, esta última registrando el mayor riesgo.

\section{DISCUSIÓN}


Este es el primer estudio caso-control realizado en el departamento del Cauca para establecer el papel de los principales factores de riesgo asociados a neoplasia cervical. El uso de un cuestionario detallado para la obtención de variables socio-demográficas, la identificación precisa de los casos y controles y el diagnóstico molecular de VPH, permitió realizar un adecuado análisis de riesgo (OR), ajustando por las potenciales variables de confusión. Los casos estuvieron caracterizados por ser mujeres, en su mayoría, procedentes de áreas rurales y con un nivel educativo bajo ( $\leq$ primaria), características poblacionales que han sido previamente descritas en la literatura como factores de riesgo para el desarrollo de lesiones intraepiteliales escamosas de alto grado (13). Estos resultados resaltan la necesidad de mejorar los programas de tamizaje en las áreas rurales que permita la detección temprana y el tratamiento oportuno de las lesiones precursoras del cáncer cervicouterino.

El presente estudio muestra que la infección con VPH es un factor de riesgo importante para el desarrollo de lesiones intraepiteliales escamosas de alto grado en el departamento del Cauca, ocurriendo en $91 \%$ de los casos. Esta frecuencia esta acorde con estudios epidemiológicos recientes que indican la presencia de VPH en más del $90 \%$ de los casos $(9,12)$. De otro lado, la frecuencia de infección con VPH fue de $36 \%$ en los controles, una cifra relativamente alta en comparación con estudios realizados en áreas urbanas en Colombia (10-15 \%) (14,15), pero muy similar con otros estudios realizados en áreas rurales en Latinoamérica (25-36 \%) $(16,17)$. En consecuencia, la alta prevalencia de infección con VPH en la población podría ser una de las causas de la alta incidencia de estas lesiones en la población femenina del departamento del Cauca.

Al igual que otros estudios $(16,18)$, la prevalencia de infección con VPH presentó dos picos en la población caucana, uno entre los 31-40 años de edad y otro para mayores de 50 años. Existen varias hipótesis para este comportamiento en prevalencia: el primer pico se asocia con el tiempo necesario para desarrollar neoplasia cervical, una vez se ha infectado con VPH. El segundo pico puede indicar una reactivación de una infección latente con VPH o que la posibilidad de identificación de VPH haya aumentado en la medida que los cambios atróficos posmenopáusicos ocurren en el tejido del cuello uterino (19). Estos datos no solamente señalan que se debe procurar por mantener en los programas de citología a las mujeres mayores de 50 años, sino también que es necesario realizar investigaciones para identificar las barreras logísticas y culturales que dificultan su seguimiento y para conocer las 
causas de la reactivación viral, lo cual podría estar mediado por la susceptibilidad genética en el sistema inmune (20).

Aunque varios estudios han reportado que el número de compañeros sexuales esta asociado con el riesgo de neoplasia, parece ser que este parámetro solo expresa la probabilidad de infección con VPH; convirtiéndose estadísticamente irrelevante frente a las nuevas técnicas moleculares para detectar la presencia de VPH (9). De otro lado, la edad a la primera relación sexual parece ser un factor de riesgo independiente $(9,17)$. En el presente estudio, el número de compañeros sexuales no afecto el riesgo de neoplasia cervical, mientras que haber tenido la primera relación sexual a una edad temprana fue estadísticamente significativo en los casos menores o iguales a 30 años. Lo último sugiere que existe un periodo de susceptibilidad al potencial oncogénico de VPH; se ha demostrado que el cuello uterino es más vulnerable durante la menarquia, cuando el tejido escamocolumnar sufre metaplasia y una infección con VPH podría alcanzar el tejido basal y establecer una infección persistente $(9,17)$.

En el presente estudio, se observó una fuerte asociación entre la multiparidad y el riesgo de neoplasia cervical, incluso después de controlar por las potenciales variables de confusión. La multiparidad ha sido asociada consistentemente con un mayor riesgo de neoplasia cervical; en el análisis restringido de mujeres VPH positivas, se ha reportado un incremento en el riesgo de NIC II, III y carcinoma in situ asociado con un incremento en el número de partos (21).

De otro lado, en el presente estudio, el uso de anticonceptivos orales redujo notablemente el riesgo de neoplasia; en particular, en mujeres con uso mayor a 5 años. Estos resultados son contrarios a la evidencia epidemiológica disponible, la cual indica un incremento en el riesgo de neoplasia en mujeres VPH positivas que llevan consumiendo anticonceptivos orales por largos periodos de tiempo (22). Una posible explicación para esta diferencia en el riesgo es que, en general, los casos tuvieron un nivel educativo inferior en comparación a los controles, lo cual esta asociado con un nivel socioeconómico bajo, y por lo tanto, reduce la capacidad de adquisición de anticonceptivos orales. Además, el continuo seguimiento citológico que se recomienda hacer a las mujeres que utilizan anticonceptivos orales, pudo influenciar la reducción de riesgo en las mujeres que los consumen. En cualquier instancia, el tamaño de muestra del presente estudio no tiene suficiente poder estadístico, por lo que es necesario ampliar la muestra para poder discriminar entre estas posibles explicaciones. 
La contaminación del aire a través de la combustión de la leña, ha sido asociada con el desarrollo de cáncer de pulmón y del tracto aero-digestivo superior (23). Durante la combustión de la leña se liberan monóxido de carbono, óxidos de sulfuro y nitrógeno, mezclas de hidrocarburos aromáticos policíclicos y otras partículas, los cuales han sido clasificados por la IARC como posibles carcinógenos a humanos (24). En el presente estudio, el riesgo de neoplasia cervical fue de 5,3 para mujeres entre 16-45 años de exposición al humo de leña y de 16,1 para mujeres expuestas $>45$ años (Tabla 2). Estos resultados sugieren que la exposición al humo de leña es un importante co-factor de riesgo para neoplasia cervical, particularmente en las mujeres infectadas con VPH. Estas observaciones son soportadas por un estudio realizado en Honduras, en el cual el riesgo de neoplasia cervical en mujeres VPH positivas fue dependiente del tiempo de exposición a humo de leña (25). Es posible que la exposición al humo de leña por varios años pueda tener un efecto biológico en la progresión de las lesiones pre-neoplásicas del epitelio cervical, induciendo la acumulación de daño genético a manera de aberraciones cromosómicas y mutaciones génicas, como se ha propuesto para otros carcinógenos ambientales, favoreciendo así la aparición de lesiones en las mujeres VPH positivas (10).

En conclusión, el presente estudio confirma la asociación entre VPH y el riesgo de lesiones intraepiteliales escamosas de alto grado (NIC II, NIC III y carcinoma in situ). Los datos sugieren que el inicio de relaciones sexuales a temprana edad, la multiparidad y la exposición a carcinógenos ambientales presentes en el humo de leña son importantes co-factores de riesgo para neoplasia cervical. Estos resultados brindan información valiosa a las instituciones de salud pública para desarrollar mejores programas de promoción y prevención de neoplasia cervical en la región y el país. •

Agradecimientos. Este proyecto fue financiado por el Programa de Retorno de Becarios de Colciencias, a través del Observatorio Colombiano de Ciencia y Tecnología (Código 1103-04-11087). Los autores expresan su agradecimiento a las Vicerrectorías de Investigaciones y Administrativa, a la Fundación de Apoyo y a la Facultad de Ciencias de la Salud de la Universidad del Cauca por su colaboración y apoyo logístico. También agradecen a los profesionales y pacientes del Hospital Nivel I del Bordo, Hospital Nivel I de Piendamó y Hospital Susana López de Valencia y Profamilia en Popayán, y al personal del Laboratorio de Genética Humana de la Universidad del Cauca por su participación y asistencia en el desarrollo de esta investigación. 


\section{REFERENCIAS}

1. Pisani P, Bray F, Parkin DM. Estimates of the world-wide prevalence of cancer for 25 sites in the adult population. Int J Cancer 2002; 97:72-81.

2. Arrossi S, Sankaranarayanan R, Parkin DM. Incidence and mortality of cervical cancer in Latin America. Salud Publica Mex 2003; 45:S306-14.

3. Organizacion Panamaricana de la Salud. Colombia Health Profile. En:Pan American Health Organization, ed. Health in the Americas Vol. II. Washington, D.C.: PAHO; 1998. p. 181-93.

4. Ministerio de Salud. SIVIGILA: Situación del cáncer en Colombia. Semana Epidemiológica No. 25 2000; 1-7.

5. Meisels A, Fortin R. Condylomatous lesions of the cervix and vagina. I. Cytologic patterns. Acta Cytol 1976; 20:505-9.

6. Oriel JD. Sex and cervical cancer. Genitourin Med 1988; 64:81-9.

7. Zur-Hausen H. Papillomaviruses in anogenital cancer as a model to understand the role of viruses in human cancers. Cancer Res 1989; 49:4677-81.

8. Walboomers JM, Jacobs MV, Manos MM, Bosch FX, Kummer JA, Shah KV, et al. Human papillomavirus is a necessary cause of invasive cervical cancer worldwide. J Pathol 1999; 189:12-9.

9. Bosch FX, Lorincz A, Muñoz N, Meijer CJ, Shah KV. The causal relation between human papillomavirus and cervical cancer. J Clin Pathol 2002; 55:24465.

10.Au WW, Sierra-Torres CH, Tyring SK. Acquired and genetic susceptibility to cervical cancer. Mutat Res 2003; 544:361-64.

11. Walsh PS, Metzger DA, Higuchi R. Chelex 100 as a medium for simple extraction of DNA for PCR-based typing from forensic material. Biotechniques 1991; 10:506-13.

12. Sierra-Torres CH, Au WW, Arrastia CD, Cajas-Salazar N, Robazetti SC, Payne DA, et al. Polymorphisms for chemical metabolizing genes and risk for cervical neoplasia. Environ Mol Mutagen 2003; 41:69-76.

13. Palacio-Mejia LS, Rangel-Gomez G, Hernandez-Avila M, Lazcano-Ponce E. Cervical cancer, a disease of poverty: mortality differences between urban and rural areas in Mexico. Salud Publica Mex 2003; 45 Suppl 3:S315-25.

14. Muñoz N, Bosch FX, de Sanjose S, Tafur L, Izarzugaza I, Gili M, et al. The causal link between human papillomavirus and invasive cervical cancer: a population-based case-control study in Colombia and Spain. Int J Cancer 1992; 52:743-9.

15. Molano M, Posso H, Weiderpass E, Van Den Brule AJ, Ronderos M, Franceschi $\mathrm{S}$, et al. Prevalence and determinants of HPV infection among Colombian women with normal cytology. Br J Cancer 2002; 87:324-33.

16. Herrero R, Hildesheim A, Bratti C, Sherman ME, Hutchinson M, Morales J, et al. Population-based study of human papillomavirus infection and cervical neoplasia in rural Costa Rica. J Natl Cancer Inst 2000; 92:464-74.

17. Sierra-Torres CH, Tyring SK, Au WW. Risk contribution of sexual behavior and cigarette smoking to cervical neoplasia. Int J Gynecol Cancer 2003; 13:61725. 
18. Melkert PW, Hopman E, Van Den Brule AJ, Risse EK, van Diest PJ, Bleker OP, et al. Prevalence of HPV in cytomorphologically normal cervical smears, as determined by the polymerase chain reaction, is age-dependent. Int $\mathrm{J}$ Cancer 1993; 53:919-23.

19. Bosch FX, Rohan T, Schneider A, Frazer I, Pfister H, Castellsague X, et al. Papillomavirus research update: highlights of the Barcelona HPV 2000 international papillomavirus conference. J Clin Pathol 2001; 54:163-75.

20. Dao DD, Sierra-Torres CH, Robazetti SC, de Gomez MN, Konig R, Lema C, et al. HLA-DQB1 and cervical cancer in Venezuelan women. Gynecol Oncol 2005; 96:349-54.

21. Castellsague X, Muñoz N. Cofactors in human papillomavirus carcinogenesis: role of parity, oral contraceptives, and tobacco smoking. J Natl Cancer Inst Monogr 2003; 31:20-8.

22. Villiers EM. Relationship between steroid hormone contraceptives and HPV, cervical intraepithelial neoplasia and cervical carcinoma. Int J Cancer 2003; 103:705-708.

23. Kleinerman RA, Wang Z, Wang L, Metayer C, Zhang S, Brenner AV, et al. Lung cancer and indoor exposure to coal and biomass in rural China. J Occup Environ Med 2002; 44:338-44.

24. International Agency for Research on Cancer. Monograph on the evaluation of the carcinogenic risk of chemicals to humans. IARC Sci Publ 1986; 38:1-421.

25. Velema JP, Ferrera A, Figueroa M, Bulnes R, Toro LA, de Barahona O, et al.

Burning wood in the kitchen increases the risk of cervical neoplasia in

HPV-infected women in Honduras. Int J Cancer 2002; 97:536-41. 